\title{
Nobiletin attenuates lipopolysaccharide/D-galactosamine-induced liver injury in mice by activating the Nrf 2 antioxidant pathway and subsequently inhibiting NF-кB-mediated cytokine production
}

\author{
ZHENXING HE ${ }^{1,2}$, XUANFEI LI ${ }^{1}$, HAO CHEN ${ }^{1}$, KUN HE $^{1}$, YIMING LIU ${ }^{1}$, JUNHUA GONG ${ }^{1}$ and JIANPING GONG ${ }^{1}$ \\ ${ }^{1}$ Department of Hepatobiliary Surgery, The Second Affiliated Hospital of Chongqing Medical University, \\ Chongqing 400010; ${ }^{2}$ Department of Hepatopancreatobiliary Surgery, North Sichuan Medical University, \\ Nanchong Central Hospital, Nanchong, Sichuan 637000, P.R. China
}

Received September 24, 2015; Accepted September 30, 2016

DOI: $10.3892 / \mathrm{mmr} .2016 .5943$

\begin{abstract}
Inflammation and oxidative stress serve an important role in the development of lipopolysaccharide/D-galactosamine (LPS/GalN)-induced acute liver injury. Nobiletin, which is found in high quantities in the peel of citrus fruits, is able to modulate immune responses, including inflammatory response and oxidative stress. The present study aimed to evaluate the protective effects of nobiletin on LPS/GalN-induced acute liver injury. Male C57BL/6 mice were intraperitoneally treated with nobiletin $(50,100$ and $200 \mathrm{mg} / \mathrm{kg}) 2 \mathrm{~h}$ prior to LPS/GalN injection. Liver injury was observed in the LPS/GalN group, as demonstrated by increased levels of serum hepatic enzymes and hepatic inflammatory mediators, as well as by histopathological alterations. Treatment with nobiletin reduced serum alanine aminotransferase and aspartate aminotransferase levels, improved hepatic structure, and suppressed hepatic interleukin (IL)-1 $\beta$, IL- 6 and tumor necrosis factor- $\alpha$ production $24 \mathrm{~h}$ after LPS/GalN exposure. Western blot analysis revealed that nobiletin treatment inhibited inducible nitric oxide synthase and cyclooxygenase-2 liver expression. In addition, nobiletin suppressed LPS/GalN-induced phosphorylation and degradation of inhibitor of nuclear factor $(\mathrm{NF})-\kappa \mathrm{B}(\mathrm{I} \kappa \mathrm{B}) \alpha$, as well as NF- $\kappa \mathrm{B}$ p 65 translocation into the nucleus. Nobiletin also upregulated the expression of nuclear NF-E2-related factor 2 (Nrf2) and cytoplasmic heme oxygenase-1. In conclusion, these results indicate that nobiletin serves a protective role in LPS/GalN-induced acute liver injury via activation of the Nrf2 antioxidant pathway and subsequent inhibition of $\mathrm{NF}-\kappa \mathrm{B}-\mathrm{mediated}$ cytokine production. These findings support
\end{abstract}

Correspondence to: Professor Jianping Gong, Department of Hepatobiliary Surgery, The Second Affiliated Hospital of Chongqing Medical University, 74 Lin Jiang Road, Chongqing 400010, P.R. China

E-mail: gongjianping11@126.com

Key words: nobiletin, D-galactosamine, lipopolysaccharide, Nrf2, $\mathrm{NF}-\kappa \mathrm{B}$ the potential for nobiletin as a therapeutic agent for the treatment of acute liver injury.

\section{Introduction}

Acute liver failure (ALF) is a severe condition, which is associated with a high rate of mortality resulting from excessive hepatocyte death in a short time (1). At present, there are limited effective preventative and therapeutic strategies for the treatment of this disease, short of liver transplantation $(2,3)$. Therefore, there is an urgent need to develop novel drugs to control this condition. Lipopolysaccharide (LPS)- and D-galactosamine (GalN)-induced acute liver injury in mice is a well-established animal model that may accurately represent clinical symptoms in humans (4). It is widely used to investigate the underlying mechanisms and potential therapies for ALF. GalN is a specific hepatotoxic agent that inhibits RNA and protein synthesis in hepatocytes (4). LPS induces the production of inflammatory cytokines, resulting in subsequent liver tissue injury (5). Furthermore, LPS induces Kupffer cell activation via the Toll-like receptor 4 signaling pathway, and subsequently activates nuclear factor (NF)- $\mathrm{B}$ and initiates the release of inflammatory cytokines, including interleukin (IL)-1 $\beta$, IL-6 and tumor necrosis factor (TNF)- $\alpha(6,7)$.

Nobiletin is an O-methylated flavonoid present in citrus peels, with an empirical formula of $\mathrm{C}_{21} \mathrm{H}_{22} \mathrm{O}_{8}$ and a molecular weight of 402.39 , which has been reported to possess anti-inflammatory and antioxidant properties (8-10). Previous studies have reported that nobiletin may protect against cisplatin-induced acute kidney injury by preserving renal function and restoring antioxidant status (11). Furthermore, a Citrus aurantium extract, which is rich in nobiletin and tangeretin, has been demonstrated to inhibit ethanol-induced liver injury in mice via modulation of AMP-activated protein kinase and NF-E2-related factor 2 (Nrf2)-related signals (12). In addition, nobiletin and tangeretin have been reported to suppress LPS-induced osteoclast formation and bone resorption in an experimental model of periodontitis (13). However, the effects of nobiletin on LPS/GalN-induced liver injury remain unclear. The present study aimed to determine the effects of nobiletin on LPS/GalN-induced liver injury in mice. 


\section{Materials and methods}

Animals. Male C57BL/6 mice (weight, 18-22 g; age, 6 weeks) were purchased from the Center of Experimental Animals of Chongqing Medical University (Chongqing, China). The mice were housed in an environmentally controlled room (temperature, $24 \pm 1^{\circ} \mathrm{C}$; humidity, $40-80 \%$ ) under a 12 -h dark/light cycle with free access to food and water. All animal experimentation described in the present study was performed according to the recommendations in the Guide for the Care and Use of Laboratory Animals of the National Institutes of Health. The study was approved by the committee on the Ethics of Animal Experiments of Chongqing Medical University.

Experimental design. A total of 50 mice were randomly divided into five groups: PBS control group, LPS/GalN group and nobiletin $(50,100$ and $200 \mathrm{mg} / \mathrm{kg})+\mathrm{LPS} / \mathrm{GalN}$ groups. To induce acute liver injury, the mice were intraperitoneally injected with LPS $(100 \mu \mathrm{g} / \mathrm{kg}$; Sigma-Aldrich; Merck Millipore, Darmstadt, Germany) and GalN (700 mg/kg; Sigma-Aldrich; Merck Millipore). A total of 50,100 or $200 \mathrm{mg} / \mathrm{kg}$ nobiletin (92600; Sigma-Aldrich; Merck Millipore) was administered intraperitoneally $2 \mathrm{~h}$ prior to LPS/GalN injection. The mice were sacrificed under anesthesia with sodium pentobarbital $(40 \mathrm{mg} / \mathrm{kg}$, intraperitoneally). Blood and liver tissues were subsequently collected for analysis.

Serum hepatic enzymes. Following LPS and GalN injection, all groups were fasted for $24 \mathrm{~h}$, and blood samples were collected from the eyeballs. Each blood sample was incubated for $30 \mathrm{~min}$ at room temperature, and was then centrifuged at $1,200 \times \mathrm{g}$ for $10 \mathrm{~min}$ at $4^{\circ} \mathrm{C}$. Serum alanine aminotransferase (ALT) and aspartate aminotransferase (AST) levels were analyzed using an automatic serum analyzer (7600-120; Hitachi High-Technologies Corporation, Tokyo, Japan).

ELISA assay. Liver tissue samples were homogenized in ice-cold PBS (Wuhan Boster Biological Technology Co., Ltd., Wuhan, China) and were centrifuged at 1,200 x $g$ for $20 \mathrm{~min}$ at $4^{\circ} \mathrm{C}$. Supernatants were collected and TNF- $\alpha$, IL- $1 \beta$ and IL-6 expression was analyzed using commercially available ELISA kits (cat. nos. EK0394, EK0411 and EK0527; Wuhan Boster Biological Technology Co., Ltd.) according to the manufacturer's protocols. The absorbance was measured at $450 \mathrm{~nm}$ using a microplate reader (Bio-Tek ELx800; Bio-Tek Instruments, Inc., Winooski, VT, USA).

Histological analysis. Liver tissues collected from the mice were fixed with $10 \%$ formalin (10D15B, Wuhan Boster Biological Technology Co., Ltd.) for $24 \mathrm{~h}$ at room temperature, embedded in paraffin wax and cut into 6- $\mu \mathrm{m}$ sections. For hematoxylin and eosin staining, the slides bearing tissue sections were stained with hematoxylin (Beyotime Institute of Biotechnology, Haimen, China) for $5 \mathrm{~min}$ and $1 \%$ eosin solution (Beyotime Institute of Biotechnology) for $30 \mathrm{sec}$ at room temperature. Following hematoxylin and eosin staining, the slides were observed for conventional morphological evaluation under a light microscope (Eclipse TE2000-U; Nikon
Corporation, Tokyo, Japan) and images were captured at x400 magnification.

Western blot analysis. A total of $24 \mathrm{~h}$ after LPS/GalN injection, liver tissues were harvested and homogenized in radioimmunoprecipitation assay lysis buffer (P0013D; Beyotime Institute of Biotechnology) to prepare whole protein extracts. Tissue lysates were centrifuged at $12,000 \times \mathrm{g}$ for $10 \mathrm{~min}$ at $4^{\circ} \mathrm{C}$. A nuclear/cytoplasmic protein extraction kit (P0028; Beyotime Institute of Biotechnology) was used to extract nuclear proteins in accordance with the manufacturer's protocol. Protein concentrations were quantified by bicinchoninic acid assay (Beyotime Institute of Biotechnology) and aliquots of $50 \mu \mathrm{g}$ per sample of liver tissue homogenates or nucleus fractionation were electrophoresed. Subsequently, proteins were separated by $10 \%$ SDS-PAGE and were transferred to polyvinylidene fluoride membranes (EMD Millipore, Billerica, MA, USA). The membranes were blocked for $1 \mathrm{~h}$ at $37^{\circ} \mathrm{C}$ using bovine serum albumin (Beyotime Institute of Biotechnology) Each membrane was incubated separately with the following rabbit primary antibodies: Anti-inducible nitric oxide synthase (iNOS) (sc-8310; 1:600), anti-cyclooxygenase (COX)-2 (sc-7951; 1:600), anti-NF-kB (sc-372; 1:500), anti-phosphorylated

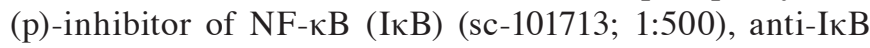
(sc-371, 1:500), anti-Nrf2 (sc-722; 1:500), anti-heme oxygenase (HO)-1 (sc-10789; 1:500), anti-GADPH (sc-25778; 1:1,000) and anti-TATA box binding protein (sc-33736; 1:1,000) (all Santa Cruz Biotechnology, Inc., Dallas, TX, USA). Subsequently, the membranes were incubated with horseradish peroxidase-linked goat anti-rabbit immunoglobulin G (1:10,000; cat. no. 7074; Cell Signaling Technology, Inc., Danvers, MA, USA) for $1 \mathrm{~h}$ at room temperature. Protein bands were visualized using a western blotting detection system (ChemiDoc ${ }^{\mathrm{TM}} \mathrm{XRS}+$; Bio-Rad Laboratories, Inc., Hercules, CA, USA) and analyzed using a densitometry system (Quantity One v4.6.2; Bio-Rad Laboratories, Inc.) according to the manufacturer's protocol.

Statistical analysis. An independent sample t-test was used to determine the statistical differences between two groups. Multiple group comparisons were tested using one-way analysis of variance, followed by Tukey post hoc test and a 95\% confidence interval $(\mathrm{P}<0.05)$ was accepted as indicative of significance. All statistical analyses were performed using SPSS 12.0 statistical software (SPSS, Inc., Chicago, IL, USA). All experiments were repeated three times and data are presented as the mean + standard error.

\section{Results}

Nobiletin inhibits LPS/GalN-induced serum ALT and AST levels. To assess the protective effects of nobiletin on LPS/GalN-induced liver injury in mice, serum ALT and AST levels were detected $24 \mathrm{~h}$ after LPS/GalN treatment. As shown in Fig. 1, ALT and AST levels were significantly increased after LPS/GalN treatment. However, the increased levels were attenuated following administration of nobiletin $(50,100$ and $200 \mathrm{mg} / \mathrm{kg})$.

LPS/GalN-induced histopathological changes are reduced by nobiletin treatment. The histopathological state of the 
A

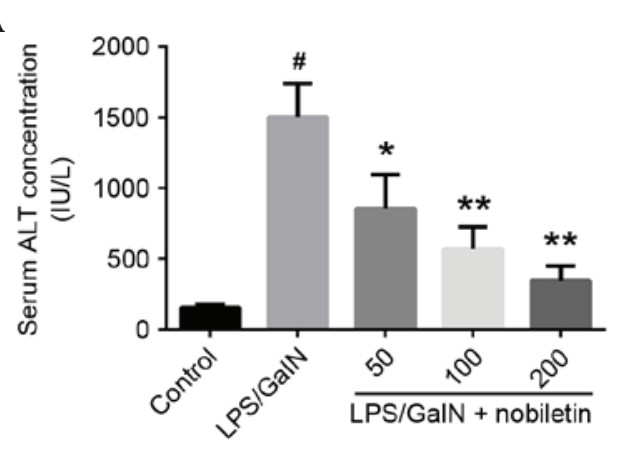

B

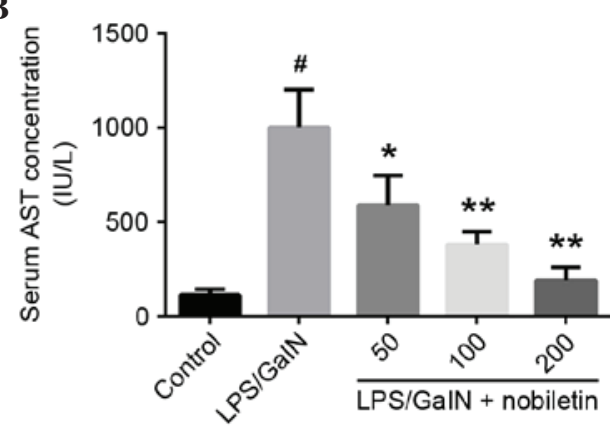

Figure 1. Effects of nobiletin on serum (A) ALT and (B) AST levels. Data are presented as the mean \pm standard error of the mean ( $\mathrm{n}=10 / \mathrm{group}) .{ }^{*} \mathrm{P}<0.01 \mathrm{vs}$. control group, "P<0.05 and ${ }^{* *} \mathrm{P}<0.01$ vs. LPS/GalN group. ALT, alanine aminotransferase; AST, aspartate aminotransferase; LPS, lipopolysaccharide; GalN, D-galactosamine.
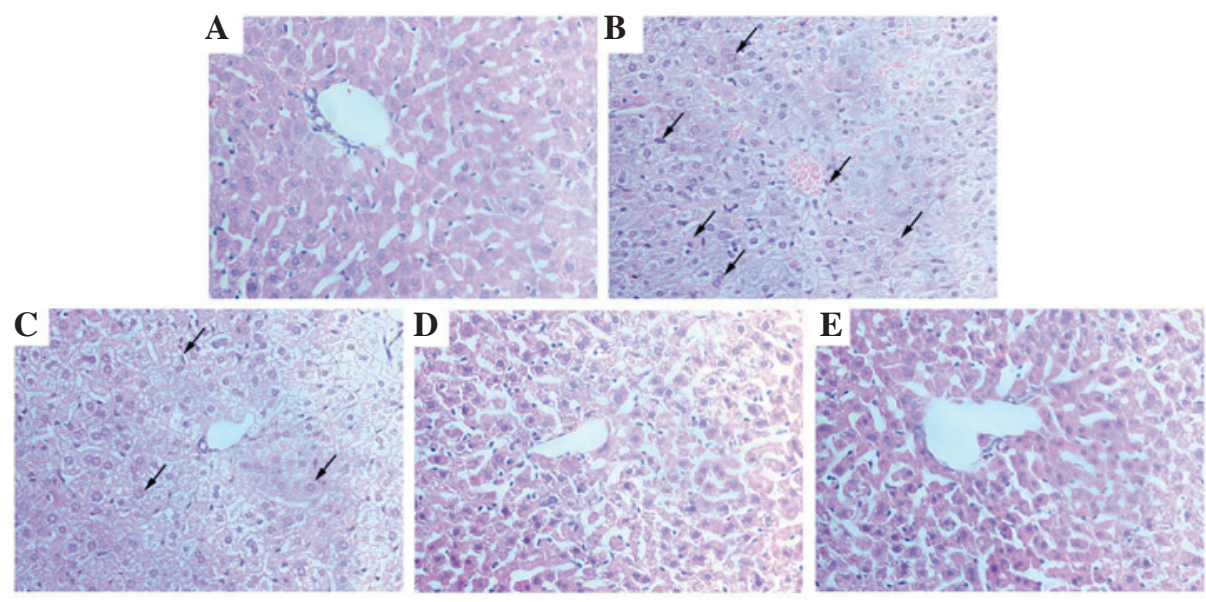

Figure 2. Effects of nobiletin on histopathological changes in liver tissues. Representative histological changes in liver tissues obtained from mice of various groups. (A) Control group, (B) LPS/GalN group, (C) LPS/GalN + nobiletin (50 mg/kg) group, (D) LPS/GalN + nobiletin (100 mg/kg) group, (E) LPS/GalN + nobiletin $(200 \mathrm{mg} / \mathrm{kg}$ ) group (hematoxylin and eosin staining, magnification 400x). LPS, lipopolysaccharide; GalN, D-galactosamine.
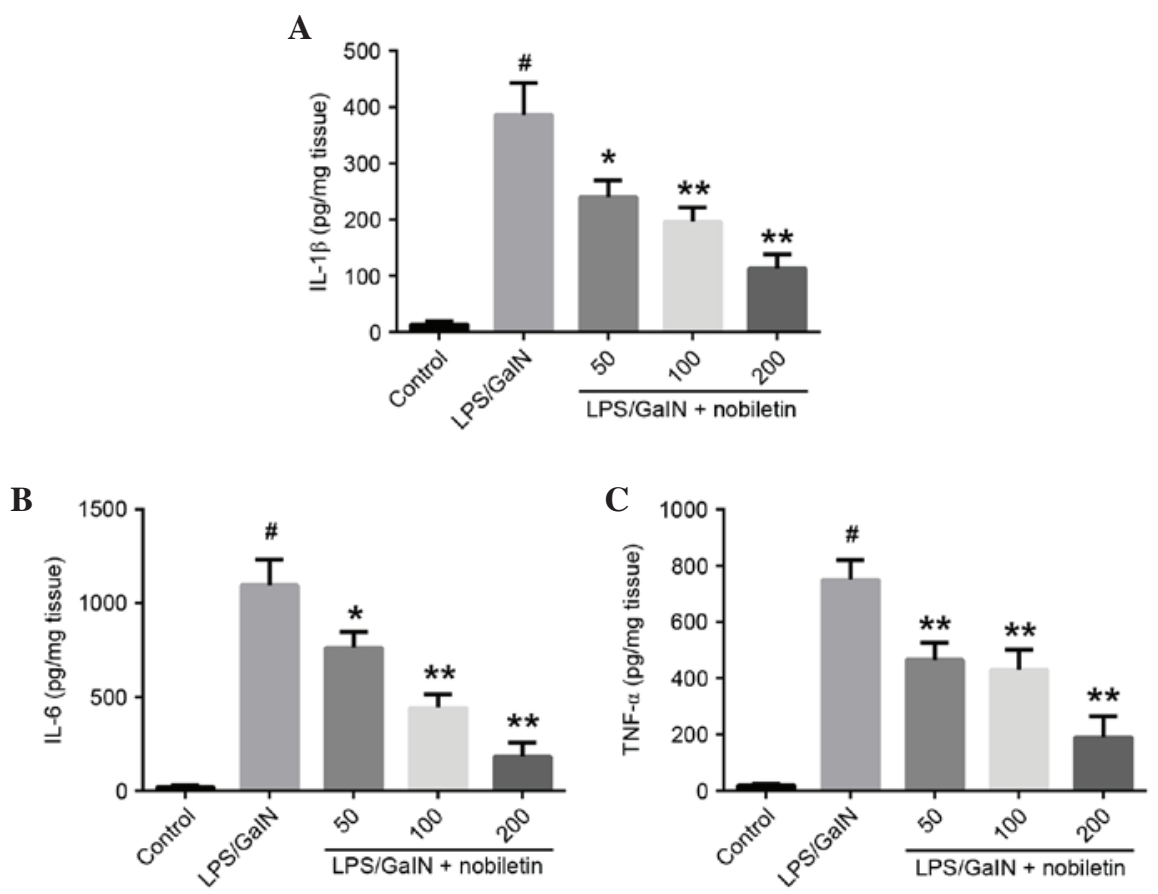

Figure 3. Effects of nobiletin on hepatic (A) IL-1 $\beta$, (B) IL-6 and (C) TNF- $\alpha$ levels. Data are presented as the mean \pm standard error of the mean ( $\mathrm{n}=10 / \mathrm{group}$ ). ${ }^{*} \mathrm{P}<0.01$ vs. control group, ${ }^{*} \mathrm{P}<0.05$ and ${ }^{* *} \mathrm{P}<0.01$ vs. LPS/GalN group. IL, interleukin, TNF- $\alpha$, tumor necrosis factor- $\alpha$; LPS, lipopolysaccharide; GalN, D-galactosamine. 

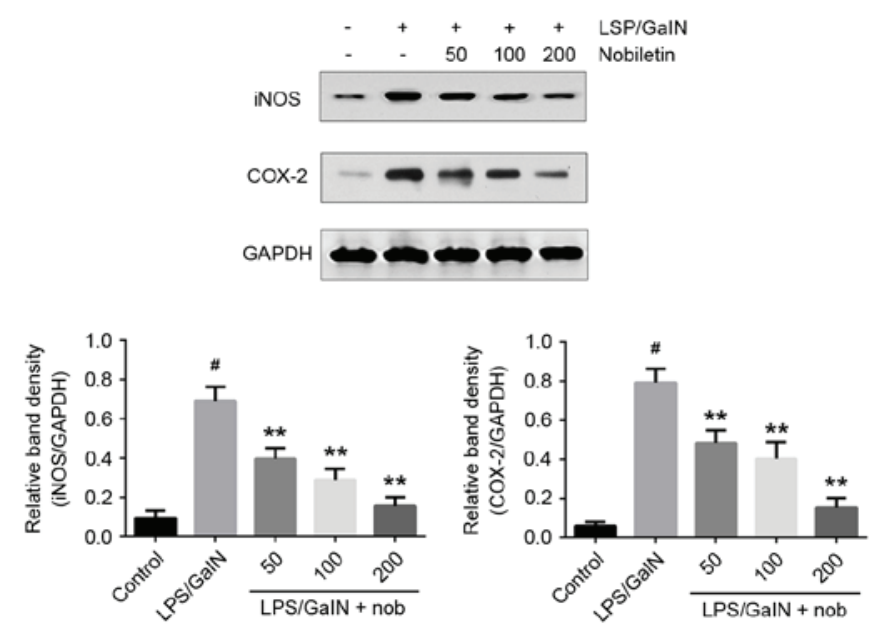

Figure 4. Effects of nobiletin on hepatic iNOS and COX-2 expression. Data are presented as the mean \pm standard error of the mean $\left(n=10 /\right.$ group). ${ }^{\#} \mathrm{P}<0.01$ vs. control group, ${ }^{* *} \mathrm{P}<0.01$ vs. LPS/GalN group. iNOS, inducible nitric oxide synthase; COX-2, cyclooxygenase-2; LPS, lipopolysaccharide; GalN, D-galactosamine.
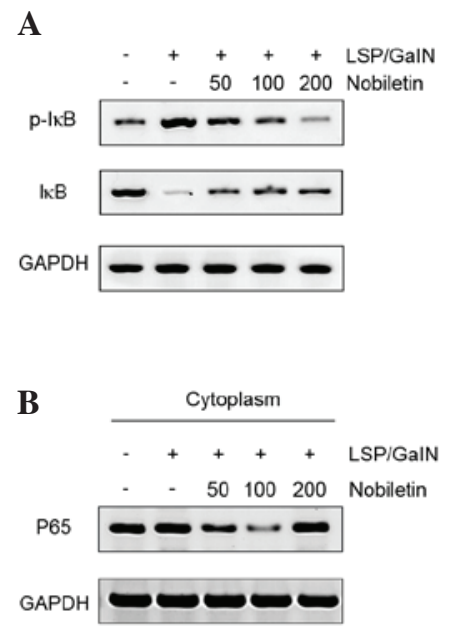
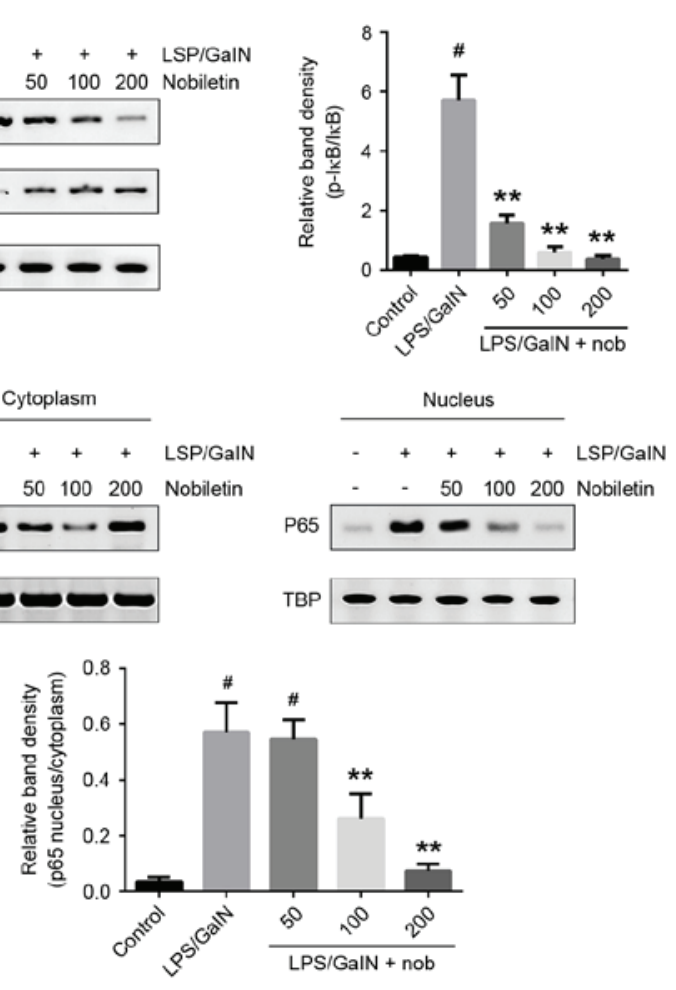

Figure 5. Nobiletin inhibits LPS/GalN-induced (A) IкB $\alpha$ phosphorylation and (B) NF- $\kappa \mathrm{B}$ activation, as determined by western blotting. Data are presented as the mean \pm standard error of the mean ( $\mathrm{n}=10$ /group). ${ }^{*} \mathrm{P}<0.01$ vs. control group, ${ }^{* *} \mathrm{P}<0.01$ vs. LPS/GalN group. I $\mathrm{B} \alpha$, inhibitor of NF- $\mathrm{B} \alpha ; \mathrm{NF}-\kappa \mathrm{B}$, nuclear factor- $\kappa \mathrm{B}$; LPS, lipopolysaccharide; GalN, D-galactosamine.

liver tissues was observed under a light microscope. As shown in Fig. 2, liver sections from the normal control group exhibited normal lobular architecture and cellular structure. Liver sections from the LPS/GalN group exhibited marked pathological alterations, including extensive areas of portal inflammation, cellular necrosis, and inflammatory cell infiltration. However, these pathological alterations were markedly attenuated in the nobiletin-treated groups.
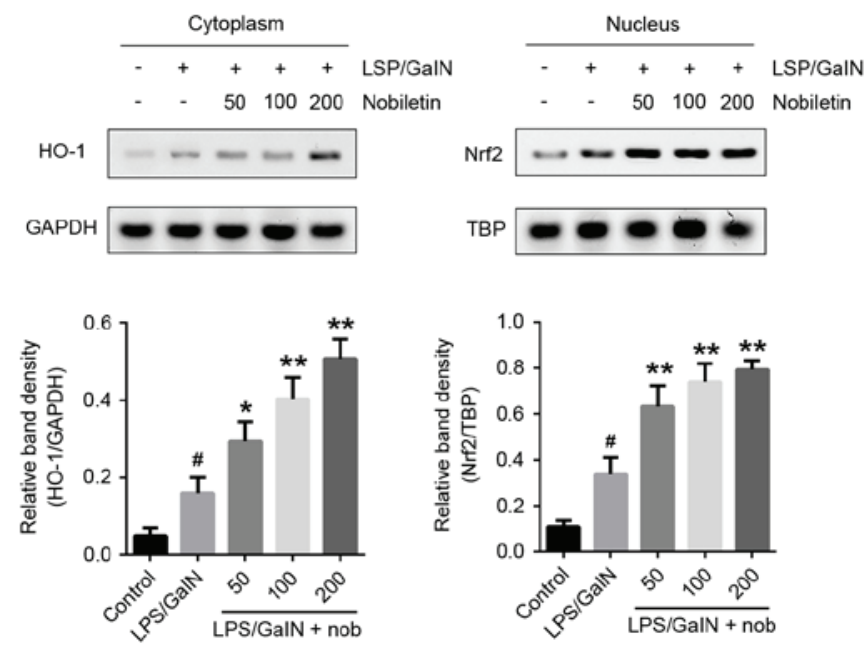

Figure 6. Nobiletin augmented the expression of Nrf2 and HO-1. Data are presented as the mean \pm standard error of the mean ( $\mathrm{n}=10$ /group). ${ }^{\#} \mathrm{P}<0.01$ vs control group, ${ }^{*} \mathrm{P}<0.05$ and ${ }^{* *} \mathrm{P}<0.01$ vs. LPS/GalN group. HO-1, heme oxygenase-1; Nrf2, NF-E2-related factor 2; TBP, Tata box binding protein; LPS, lipopolysaccharide; GalN, D-galactosamine.

Nobiletin inhibits hepatic IL-1 $\beta, I L-6$ and TNF- $\alpha$ production. To determine the anti-inflammatory effects of nobiletin, hepatic IL-1 $\beta$, IL- 6 and TNF- $\alpha$ production was detected using ELISA kits. As presented in Fig. 3, IL-1 $\beta$, IL-6 and TNF- $\alpha$ levels were significantly increased in the LPS/GalN group compared with in the control group. Nobiletin treatment $(50,100$ and $200 \mathrm{mg} / \mathrm{kg}$ ) significantly suppressed LPS/GalN-induced hepatic IL-1 $\beta$, IL- 6 and TNF- $\alpha$ levels in a dose-dependent manner.

Nobiletin inhibits hepatic iNOS and COX-2 expression. Hepatic iNOS and COX-2 expression levels were detected $24 \mathrm{~h}$ after LPS/GalN treatment by western blot analysis. The results indicated that iNOS and COX-2 levels were significantly increased in the LPS/GalN group compared with in the control group. Treatment with nobiletin (50, 100 and $200 \mathrm{mg} / \mathrm{kg}$ ) dose-dependently reduced hepatic iNOS and COX-2 levels (Fig. 4).

Nobiletin inhibits $N F-\kappa B$ activation. NF- $\kappa$ B has been reported to have a dominant role in the inflammatory response (14). In the present study, the effects of nobiletin on the degradation of $\mathrm{I} \kappa \mathrm{B} \alpha$ and the activation of $\mathrm{NF}-\kappa \mathrm{B}$ were detected by western blot analysis. The results revealed that LPS/GalN exposure induced phosphorylation and degradation of IкB $\alpha$, and NF- $\kappa$ B p65 translocation into the nucleus; however, these effects were attenuated following nobiletin treatment (Fig. 5).

Nobiletin promotes the expression of $\mathrm{Nrf} 2$ and $\mathrm{HO}-1$. Nrf2 is considered an important transcription factor in regulation of the antioxidant response (15). In the present study, the effects of nobiletin were detected on $\mathrm{Nrf} 2$ and $\mathrm{HO}-1$ expression by western blot analysis. As shown in Fig. 6, the expression levels of Nrf2 in the nucleus and HO-1 in the cytoplasm were significantly increased $24 \mathrm{~h}$ after LPS/GalN treatment. Notably, these increases in Nrf2 and HO-1 expression were augmented by nobiletin. 


\section{Discussion}

The present study demonstrated that nobiletin exerts a protective effect on LPS/GalN-induced acute liver injury in mice. The results indicated that nobiletin treatment significantly attenuated hepatic pathological damage, hepatic enzyme release, proinflammatory cytokine production and inflammatory cell infiltration. Furthermore, the results revealed that nobiletin inhibits $\mathrm{NF}-\kappa \mathrm{B}$ activation, and upregulates the expression levels of Nrf2 and HO-1. Therefore, the present study suggests that nobiletin may be considered a promising therapeutic reagent for the treatment of acute liver injury.

ALT and AST are two transaminase enzymes that are important for the synthesis of amino acids. ALT is predominantly present in the liver, whereas AST is also present in other organs and tissues. Significantly elevated levels of ALT and AST are often associated with hepatic ailments, such as viral hepatitis, liver damage and bile duct problems; and are therefore commonly used for assessing the progression of liver disease (16). Treatment with LPS/GalN can increase hepatocyte membrane permeability and induce hepatocyte necrosis, which may increase the levels of AST and ALT. In the present study, serum ALT and AST levels, and histological analysis of liver tissues, were used to assess the protective effects of nobiletin on LPS/GalN-induced acute liver injury. The results indicated that nobiletin significantly decreased serum ALT and AST levels. Furthermore, histological analysis demonstrated that nobiletin attenuated cellular swelling and necrosis in the liver, and inhibited inflammatory cell infiltration. These results suggested that nobiletin may exert a protective effect on LPS/GalN-induced hepatic injury.

Inflammation is an important pathological mechanism responsible for propagating LPS/GalN-induced liver injury (17). Previous studies have revealed that LPS may activate Kupffer cells, which can mediate the hepatic inflammatory process by producing TNF- $\alpha$, IL-1 $\beta$ and IL-6, as well as other proinflammatory cytokines $(18,19)$. TNF- $\alpha$ is an important inflammatory mediators involved in LPS/GalN-induced liver injury, which can potentially induce apoptosis of hepatocytes, subsequently leading to organ failure (20-22). In addition, TNF- $\alpha$ may initiate the inflammatory cascade and induce the production of other cytokines, including IL-1 $\beta$ and IL-6 (23). Previous studies have reported that inhibition of TNF- $\alpha$ synthesis inhibits cytokine production and attenuates liver injury (24). In addition, iNOS and COX-2 are important proinflammatory enzymes involved in the development of liver injury $(25,26)$. Elevated iNOS and COX-2 levels are often observed in LPS/GalN-induced liver injury in mice (27). In the present study, nobiletin significantly inhibited LPS/GalN-induced increases in hepatic TNF- $\alpha$, IL-1 $\beta$, IL-6, COX-2 and iNOS levels.

$\mathrm{NF}-\kappa \mathrm{B}$ is an upstream regulator of various genes associated with the inflammatory response (28). Once activated by

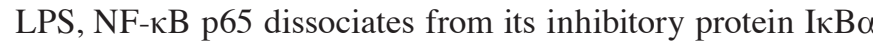
and translocates from the cytoplasm to the nucleus, where it may induce transcription of inflammatory mediators, such as TNF- $\alpha$, COX-2 and iNOS $(29,30)$. Therefore, the present study assessed the effects of nobiletin on the NF- $\mathrm{B}$ signaling pathway. The results indicated that LPS/GalN induced the phosphorylation and degradation of $\mathrm{I} \kappa \mathrm{B} \alpha$ and the nuclear translocation of $\mathrm{NF}-\kappa \mathrm{B}$ p 65 ; however, these effects were attenuated by nobiletin.

Nrf2 is an important transcription factor involved in regulating the oxidative stress response $(31,32)$. It regulates the transcription of antioxidant and detoxification genes, such as HO-1 $(33,34)$. In the present study, the expression levels of $\mathrm{Nrf} 2$ in the nucleus and HO-1 in the cytoplasm were significantly increased in the liver tissue of LPS/GalN-treated mice. Notably, these increases were augmented by nobiletin. These results suggested that nobiletin may alleviate LPS/GalN-induced oxidative damage by stimulating the oxidative defense system. Furthermore, several studies have reported that there is an interaction between the Nrf2 and $N F-\kappa B$ signaling pathways $(35,36)$. Nrf2 can negatively regulate the $\mathrm{NF}-\kappa \mathrm{B}$ signaling pathway and ameliorate $\mathrm{NF}-\kappa \mathrm{B}$-related inflammatory response and oxidative injury in mice (37). Therefore, the inhibition of NF- $\kappa \mathrm{B}$ observed in the liver tissue may be due to activation of $\mathrm{Nrf} 2$ by nobiletin treatment.

In conclusion, the present study demonstrated that treatment with nobiletin reduces LPS/GalN-induced proinflammatory cytokine production and attenuates liver injury in a mouse model. This protective mechanism may be dependent on the inhibition of $\mathrm{NF}-\kappa \mathrm{B}$ activation and the activation of Nrf2. These findings support the potential use of nobiletin as a therapeutic agent for the prevention of acute liver injury.

\section{References}

1. Pierre PG, Solbreux P and Vaerman JP: Cholera toxin-induced fluid secretion in rat gut ligated loops: Influence of bile from normal or cholera toxin-immunized rats. Immunology 68: 319-324, 1989.

2. Forbes SJ, Gupta S and Dhawan A: Cell therapy for liver disease: From liver transplantation to cell factory. J Hepatol 62 (Suppl 1): S157-S169, 2015

3. Bernal W, Lee WM, Wendon J, Larsen FS and Williams R: Acute liver failure: A curable disease by 2024? J Hepatol 62 (Suppl 1): S112-S120, 2015.

4. Kuroda N,Inoue K,Ikeda T,Hara Y, Wake K and Sato T: Apoptotic response through a high mobility box 1 protein-dependent mechanism in LPS/GalN-induced mouse liver failure and glycyrrhizin-mediated inhibition. PLoS One 9: e92884, 2014

5. Shukla P, Rao GM, Pandey G, Sharma S, Mittapelly N, Shegokar R and Mishra PR: Therapeutic interventions in sepsis: Current and anticipated pharmacological agents. Br J Pharmacol 171: 5011-5031, 2014.

6. Tsutsui $\mathrm{H}$ and Nishiguchi S: Importance of Kupffer cells in the development of acute liver injuries in mice. Int J Mol Sci 15: 7711-7730, 2014.

7. Moratalla A, Caparrós E, Juanola O, Portune K, Puig-Kröger A, Estrada-Capetillo L, Bellot P, Gómez-Hurtado I, Piñero P, Zapater P, et al: Bifidobacterium pseudocatenulatum CECT7765 induces an M2 anti-inflammatory transition in macrophages from patients with cirrhosis. J Hepatol 64: 135-145, 2015.

8. Nakajima A, Ohizumi Y and Yamada K: Anti-dementia activity of nobiletin, a citrus flavonoid: A review of animal studies. Clin Psychopharmacol Neurosci 12: 75-82, 2014.

9. Xiu LJ, Sun DZ, Jiao JP, Yan B, Qin ZF, Liu X, Wei PK and Yue XQ: Anticancer effects of traditional Chinese herbs with phlegm-eliminating properties-An overview. J Ethnopharmacol 172: 155-161, 2015.

10. Horigome S, Yoshida I, Tsuda A, Harada T, Yamaguchi A, Yamazaki K, Inohana S, Isagawa S, Kibune N, Satoyama T, et al: Identification and evaluation of anti-inflammatory compounds from Kaempferia parviflora. Biosci Biotechnol Biochem 78: 851-860, 2014

11. Malik S, Bhatia J, Suchal K, Gamad N, Dinda AK, Gupta YK and Arya DS: Nobiletin ameliorates cisplatin-induced acute kidney injury due to its anti-oxidant, anti-inflammatory and anti-apoptotic effects. Exp Toxicol Pathol 67: 427-433, 2015. 
12. Choi BK, Kim TW, Lee DR, Jung WH, Lim JH, Jung JY, Yang SH and Suh JW: A polymethoxy flavonoids-rich Citrus aurantium extract ameliorates ethanol-induced liver injury through modulation of AMPK and Nrf2-related signals in a binge drinking mouse model. Phytother Res 29: 1577-1584, 2015.

13. Tominari T, Hirata M, Matsumoto $\mathrm{C}$, Inada $\mathrm{M}$ and Miyaura $\mathrm{C}$ : Polymethoxy flavonoids, nobiletin and tangeretin, prevent lipopolysaccharide-induced inflammatory bone loss in an experimental model for periodontitis. J Pharmacol Sci 119: 390-394, 2012.

14. Mair F, Joller S, Hoeppli R, Onder L, Hahn M, Ludewig B, Waisman A and Becher B: The NF kappa B-inducing kinase is essential for the developmental programming of skin-resident and IL-17-producing gamma delta T cells. eLife 4: e10087, 2015.

15. Lu MC, Ji JA, Jiang ZY and You QD: The Keap1-Nrf2-ARE Pathway As a Potential Preventive and Therapeutic Target: An Update. Med Res Rev 36: 924-963, 2016.

16. van Beek JH, de Moor MH, de Geus EJ, Lubke GH, Vink JM, Willemsen $\mathrm{G}$ and Boomsma DI: The genetic architecture of liver enzyme levels: GGT, ALT and AST. Behav Genet 43: 329-339, 2013.

17. Liang DY, Liu LM, Ye CG, Zhao L, Yu FP, Gao DY, Wang YY, Yang ZW and Wang YY: Inhibition of UII/UTR system relieves acute inflammation of liver through preventing activation of NF-kappaB pathway in ALF mice. PLoS One 8: e64895, 2014

18. Liu LM, Liang DY, Ye CG, Tu WJ and Zhu T: The UII/UT system mediates upregulation of proinflammatory cytokines through p38 MAPK and NF- $\mathrm{B}$ pathways in LPS-stimulated Kupffer cells. PLoS One 10: e0121383, 2015.

19. Yang P, Zhou W, Li C, Zhang M, Jiang Y, Jiang R, Ba H, Li C, Wang J, Yin B, et al: Kupffer-cell-expressed transmembrane TNF- $\alpha$ is a major contributor to lipopolysaccharide and D-galactosamine-induced liver injury. Cell Tissue Res 363 371-383, 2016

20. Ahmad A, Raish M, Ganaie MA, Ahmad SR, Mohsin K, Al-Jenoobi FI, Al-Mohizea AM and Alkharfy KM: Hepatoprotective effect of Commiphora myrrha against d-GalN/LPS-induced hepatic injury in a rat model through attenuation of pro inflammatory cytokines and related genes. Pharm Biol 53: 1759-1767, 2015.

21. Ma L, Gong X, Kuang G, Jiang R, Chen R and Wan J: Sesamin ameliorates lipopolysaccharide/d-galactosamine-induced fulminant hepatic failure by suppression of Toll-like receptor 4 signaling in mice. Biochem Biophys Res Commun 461: 230-236, 2015.

22. Yamada Y, Ishizaki M, Kido T, Honda R, Tsuritani I, Nogawa K and Yamaya H: Relationship between serum gamma-glutamyl transpeptidase activity, blood pressure and alcohol consumption. J Hum Hypertens 3: 409-417, 1989.
23. Cordero-Coma M and Sobrin L: Anti-tumor necrosis factor-alpha therapy in uveitis. Surv Ophthalmol 60: 575-589, 2015.

24. Ma Z, Hou T, Shi W, Liu W and He H: Inhibition of hepatocyte apoptosis: An important mechanism of corn peptides attenuating liver injury induced by ethanol. Int J Mol Sci 16: 22062-22080, 2015.

25. Peixoto CA and Gomes FO: The role of phosphodiesterase-5 inhibitors in prostatic inflammation: A review. J Inflamm (Lond) 12: 54, 2015.

26. Sun J, Zhang S, Zhang X, Zhang X, Dong H and Qian Y: IL-17A is implicated in lipopolysaccharide-induced neuroinflammation and cognitive impairment in aged rats via microglial activation. J Neuroinflammation 12: 165, 2015.

27. Li J, Zhang $\mathrm{X}$ and Huang $\mathrm{H}$ : Protective effect of linalool against lipopolysaccharide/D-galactosamine-induced liver injury in mice. Int Immunopharmacol 23: 523-529, 2014.

28. Senegas A, Gautheron J, Maurin AG and Courtois G: IKK-related genetic diseases: Probing NF- $\kappa$ B functions in humans and other matters. Cell Mol Life Sci 72: 1275-1287, 2015.

29. Verstrepen L and Beyaert R: Receptor proximal kinases in $\mathrm{NF}-\kappa \mathrm{B}$ signaling as potential therapeutic targets in cancer and inflammation. Biochem Pharmacol 92: 519-529, 2014.

30. Muriach M, Flores-Bellver M, Romero FJ and Barcia JM: Diabetes and the brain: Oxidative stress, inflammation, and autophagy. Oxid Med Cell Longev 2014: 102158, 2014.

31. Dodson M, Redmann M, Rajasekaran NS, Darley-Usmar V and Zhang J: KEAP1-NRF2 signalling and autophagy in protection against oxidative and reductive proteotoxicity. Biochem J 469: 347-355, 2015.

32. Jiménez-Osorio AS, González-Reyes S and Pedraza-Chaverri J: Natural Nrf2 activators in diabetes. Clin Chim Acta 448 182-192, 2015.

33. Hahn ME, Timme-Laragy AR, Karchner SI and Stegeman JJ: Nrf2 and Nrf2-related proteins in development and developmental toxicity: Insights from studies in zebrafish (Danio rerio). Free Radic Biol Med 88: 275-289, 2015.

34. Kanninen KM, Pomeshchik Y, Leinonen H, Malm T, Koistinaho J and Levonen AL: Applications of the Keap1-Nrf2 system for gene and cell therapy. Free Radic Biol Med 88: 350-361, 2015.

35. Buelna-Chontal M and Zazueta C: Redox activation of Nrf2 \& NF-кB: A double end sword? Cell Signal 25: 2548-2557, 2013.

36. Agca CA, Tuzcu M, Hayirli A and Sahin K: Taurine ameliorates neuropathy via regulating $\mathrm{NF}-\kappa \mathrm{B}$ and $\mathrm{Nrf} 2 / \mathrm{HO}-1$ signaling cascades in diabetic rats. Food Chem Toxicol 71: 116-121, 2014.

37. Jiang T, Tian F, Zheng H, Whitman SA, Lin Y, Zhang Z, Zhang N and Zhang DD: Nrf2 suppresses lupus nephritis through inhibition of oxidative injury and the NF- $\mathrm{BB}$-mediated inflammatory response. Kidney Int 85: 333-343, 2014. 